\title{
1 The genetic and social contributions to sex differences in lifespan in Drosophila serrata
}

3 Running title: Sex differences in lifespan

4

5 Vikram P. Narayan ${ }^{1,2}$, Alastair J. Wilson ${ }^{2}$, Stephen F. Chenoweth ${ }^{1 *}$

6

7

1. The School of Biological Sciences, The University of Queensland, St. Lucia, Qld 4072, Australia

2. College of Life and Environmental Sciences, University of Exeter, Penryn, UK

14 Corresponding author: s.chenoweth@uq.edu.au

15

16 Word count: 2904 


\section{Abstract}

19 Sex differences in lifespan remain an intriguing puzzle for evolutionary biologists. A possible

20 explanation for lower lifespan in males is the unconditional expression of recessive deleterious

21 alleles in heterogametic $\mathrm{X}$ chromosomes in males (the unguarded $\mathrm{X}$ hypothesis). Empirical

22 evidence, however, has yielded controversial results that can be attributed to differences in both

23 genetic and social background. Here, we test the unguarded X hypothesis in Drosophila serrata

24 using a factorial design to quantify the effects of genotype, sex, social environment, and their 25 interactions on phenotypic variation for lifespan. Using an experimental approach, we 26 manipulated two inbred laboratory genotypes and their reciprocal F1s, while controlling for 27 different levels of density and mating status to account for any potential social effects. Our 28 results also show subtle but significant genotype dependent effects for both density and mating, 29 but ultimately find the unguarded $\mathrm{X}$ hypothesis insufficient to fully explain sexual dimorphism 30 in D. serrata lifespan.

32 Keywords: ageing, lifespan, Drosophila, sex, genetic, environment, unguarded X hypothesis 


\section{Introduction}

The question of why males and females differ in lifespan has long fascinated evolutionary biologists. While exceptions exist, across many taxa it is most often females that live longer than males (Austad, 2019). Despite a long history of ageing research, no proven or unifying theories have emerged, and studies still yield contradictory results. Sexual dimorphism in lifespan can arise in response to sex differences in selection on life histories. Males and females maximise reproductive fitness in different ways (Friberg, 2005, Maklakov et al., 2009) with males typically investing more in early reproduction than females, even at the cost of their own somatic maintenance and lifespan (Maklakov and Lummaa, 2013). Selection therefore alters the overall costs of reproduction for each sex, and affects the evolution of ageing by shaping sex-specific mortality rates (Promislow 2003; Bonduriansky et al. 2008). Sexual dimorphism in lifespan may also be caused by asymmetric inheritance of uneven numbers of sex chromosomes between males and females. This hypothesis posits that for species where males are the hemizygous sex, harmful recessive mutations on the $\mathrm{X}$ chromosome will always be expressed in males whereas they will commonly be masked by dominance in females (Trivers, 1985). A general prediction of this hypothesis coined the "unguarded X hypothesis" is that males should therefore on average have shorter lifespans than females.

Several studies have shown that variation in environmental or genetic background, can influence sexual dimorphism in lifespan (Kimber and Chippindale, 2013, Brengdahl et al., 2018b, Sultanova et al., 2018). Species of the genus Drosophila have featured prominently in aging research. In addition to D. melanogaster [see reviews by (Rogina, 2011) and (Piper and Partridge, 2018)], other species such as D. simulans (Ballard, 2005) have also been used as models for aging research. With the development of the Drosophila serrata Genome Reference Panel, a panel of re-sequenced lines (DsGRP) (Reddiex et al., 2018), D. serrata has now also emerged as a potential model for aging research. Here, we describe the results of a systematic analysis of lifespan comparisons in two highly inbred laboratory wild-type strains: DsGRP20 and DsGRP57. Using inbred lines can provide insight into how the underlying genetic architecture of lifespan varies in response to genetic and social conditions. For instance, Swindell and Bouzat (2006) showed that stressful environments such as increased competition and temperature had pronounced effects on mitigating lifespan reducing effects of inbreeding depression in D. melanogaster. While the existence of inbreeding depression on lifespan are 

et al., 2013, Brengdahl et al., 2018b, Sultanova and Carazo, 2019) such as mating and density is less well understood.

For D. melanogaster, the few studies where organismal condition was manipulated and lifespan was measured, both male- and female-biased effects on lifespan were found. This was true for genetic and environmental manipulations of condition. These studies highlight the importance of not just different genotypes, but also how sex differences in mating costs and behaviour affect survival rates (Burger and Promislow, 2004). This substantial variation in male and female responses emphasizes the importance of including not only both sexes, but also their social environment when analysing lifespan. Amongst the different social effects that have an impact on adult lifespan, mating activity and adult population density have been shown to influence longevity (Malick and Kidwell, 1966, Iliadi et al., 2009). In species of Drosophila, such as $D$. virilis, mating status significantly affected fly lifespan, with male and female virgins being affected very differently (Aigaki and Ohba, 1984). In D. virillis male sexual activity played the most important role amongst the complex interactions between both sexes. Mating status also affected the lifespan of both female and male $D$. melanogaster flies, though males were less affected (Koliada et al., 2020). The few systematic studies conducted on effects of high adult density, have found increased male sensitivity to variations in density, erratic mortality rates, and decreased mortality among higher density cohorts of middle-aged $D$. melanogaster females (Khazaeli et al., 1996).

This study aims to clarify how genetic background, sex, inbreeding, mating, and density act and interact with each other to shape lifespan in Drosophila serrata. In doing this we test the specific predictions of unguarded $X$ and evaluate their sensitivity to genetic and social backgrounds. To quantify effects of genotype on lifespan we crossed fully inbred flies to generate outbred and reciprocal F1 flies (Vaiserman et al., 2013). To explore interactions with social contexts of mating (Aigaki and Ohba, 1984, Service, 1989, Zajitschek et al., 2013), we measured the lifespan of these flies as both virgins and non-virgins. Furthermore, we varied the population density of flies held together in a vial, as this is also known to affect lifespan and mortality rates (Graves and Mueller, 1993, Khazaeli et al., 1995, Khazaeli et al., 1996, Joshi and Mueller, 1997). This will ultimately bring us closer in our attempts to characterise sexual dimorphism in lifespan resulting from sex differences in selection as opposed to 
present evidence of genetic interactions with sex and also with mating and density on survival characteristics in D. serrata.

\section{Materials and Methods}

\section{Fly stocks and culturing conditions}

All analyses were carried out using fruit fly genotypes, DsGRP20 and DsGRP57, randomly chosen from the DsGRP (Reddiex et al., 2018). Flies were maintained in vials containing agarsugar-yeast medium, in a temperature-controlled room at $25^{\circ} \mathrm{C}$ and a $12 / 12 \mathrm{~h} \mathrm{light/dark} \mathrm{cycle.}$ We then performed density-controlled crosses between the two lines to produce inbred

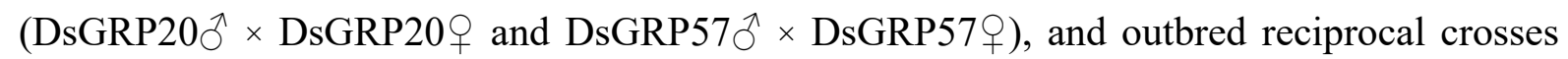

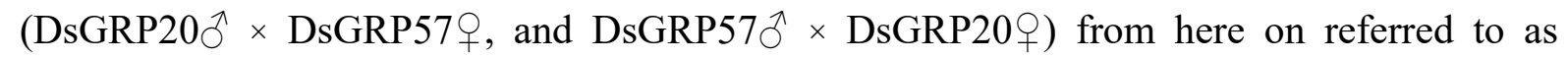
genotypes. All experimental flies were collected as virgins within $6 \mathrm{~h}$ after eclosion, and male and female offspring from each cross were randomly allocated into the experimental treatments in a factorial design including the effects of mating, and density. Flies in the mated treatment were allowed to mate for 2 days, collected using $\mathrm{CO}_{2}$, sorted by sex, and transferred to experimental vials for the lifespan trial. For each cross, virgin and mated treatments were maintained at three different vial densities. Vial densities were 5, 10 and 15 flies per vial (10 replicate vials per variant, per sex).

\section{Lifespan assay}

Vials were randomized and flies tipped into fresh food vials without anesthesia every 3-4 days. On these occasions, dead flies were counted and removed to prevent them from being tipped into the fresh food vials. Survivorship was scored at the time of tipping until all flies had died. Flies that escaped while tipping were censored. Thus, for each specific combination of genotype, sex, mating, and density the minimum number of flies was 50 and the total number of flies was 4800 before censoring. This factorial design enables us to quantify the effects of genotype, sex, social environment, and their interactions on phenotypic variation for lifespan.

\section{Statistical analyses}

To compare the effects of sex, genotype, mating, and density on adult lifespan, we used a mixed model analysis of variance using restricted maximum likelihood (REML) estimates of the variance components (PROC GLIMMIX in SAS 9.2). Sex, DsGRP genotypes (DsGRP20 ơ × 


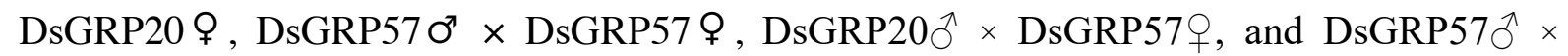

133 DsGRP20 9 ,), mated status (non-mated/mated), density (5, 10 and 15 flies per vial) and their 134 interactions were modelled as fixed factors and tested with F-statistics. For tests of fixed 135 effects, we applied a Satterthwaite approximation to calculate the denominator degrees of 136 freedom via the "ddfm=SAT" option in SAS. Vial was modelled as a random effect. Density 137 was treated as a categorical factor as we did not necessarily expect linear relationships between 138 density and longevity. Models were simplified by backward single term deletions ( $\mathrm{p} \leq 0.05)$.

139 Significant interactions that included sex were explored by fitting the mixed model separately 140 for each sex.

142 In our initial modelling, we used a four-level 'genotype' effect that includes the homozygous 143 founder lines (DsGRP20 and DsGRP57) and both reciprocal F1 crosses between these lines. 144 Subsequent contrasts between these four levels allowed us to test multiple genetic effects. First, 145 we compared homozygous line differences to assess genetic differences in lifespan. Second, 146 contrasts between the F1 and homozygous genotypes permitted a test for the effect of 147 inbreeding. Third, contrasts between the two F1 crosses allowed us to test for a reciprocal cross 148 effect that includes X chromosome genome influences. We present effect sizes as least square means and used Tukey's HSD to correct for multiple testing. 


\section{Results}

152 After censoring 194 flies that escaped while being transferred to fresh holding vials $(<5 \%$ of total flies), 4606 flies were available for analysis. Across the entire experiment, female-biased longevity was apparent. While female $D$. serrata lived on average 54 days (range 4 - 104 days), males lived an average of only 34 days (range 4-69 days). The final simplified linear model describing genetic and environmental influences on lifespan variation appears in Table 1. While the model provided statistical support for sex differences in lifespan in D. serrata (Sex: $\left.F_{1,454.4}=1798.3, P=4.54^{\mathrm{e}-160}\right)$, males and females were influenced differently by genotype (Sex $x$ Genotype: $F_{3,454.3}=64.6, P=8.36^{\mathrm{e}-35}$ ), which was also a significant main effect in the model (Genotype: $F_{3,601.3}=340.4, P=3.83^{\mathrm{e}-129}$ ). Here, three key results are of interest. First, reciprocal crossing did not affect the degree of sexual dimorphism with no lifespan differences found

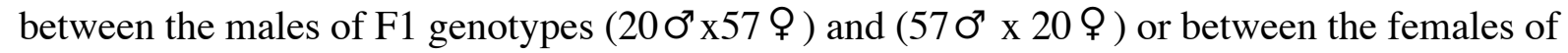
these two F1 genotypes (Fig. 1). Second, males and females were affected by outcrossing in different ways. F1 females lived at least 17 days longer than homozygous parental line females and a similar degree of increase $(\sim 40 \%)$ was observed in F1 males compared to parental line DsGRP20 males (Fig. 1). However, there was no difference in male lifespan between the F1s and parental line DsGRP57 (Fig. 1) consistent with a lack of any outcrossing effect. Third, genetic differences were also apparent between the two parental lines with both males and females from line DsGRP57 living between 14 and 7 days longer than males and females from line DsGRP20 respectively.

172 Our analysis also indicated a genotype-by-environment interaction for lifespan. Genotype 173 dependent effects were observed for both density and mating via a significant three-way interaction (Table 1: Genotype $\times$ Density $\times$ Mating: $F_{6,552.8}=2.45, P=0.024$ ). The social environmental effects underlying this significant interaction were, however, typically more subtle than the effects seen in the interaction between sex and genotype (Fig. 2) Considering this interaction further, post-hoc comparisons revealed significant differences between density and mating within only two of the four genotypes the parental ( $570^{7} \times 57$ O $)$ and the reciprocal

180 density treatments, with the lifespan of mated flies on average, 6 days higher than unmated flies (Fig. 2). 


\section{Discussion}

187 All treatment combinations female D. serrata lived longer than males, a result consistent with a wide range of wild and captive species, where on average, the homogametic sex lives longer than its heterogametic counterpart (Xirocostas et al., 2020). Our result is also consistent with two previous studies of Drosophila serrata both of which indicate female-biased longevity (Robson et al., 2006, Wit et al., 2015). One prominent hypothesis for reduced male lifespan is the "unguarded X" hypothesis (Trivers, 1985). This hypothesis predicts that reduced male lifespan is a result of the unconditional expression of recessive deleterious alleles on the single $\mathrm{X}$ chromosome. To date, the few studies that have explicitly tested predictions arising from the unguarded X hypothesis, conducted in Drosophila melanogaster (Carazo et al., 2016, Sultanova et al., 2018, Brengdahl et al., 2018a) have produced inconsistent results.

Here, we used two inbred lines with differing lifespans to create outbred and reciprocal F1's to test for reduced lifespan in males as predicted by the unguarded $\mathrm{X}$ hypothesis. Despite differences in inbred parental lifespan, we found no differences in lifespan between the outbred and reciprocal male F1's that could be attributed to the accumulation of recessive deleterious mutations on the $\mathrm{X}$ chromosome as predicted by the unguarded $\mathrm{X}$ hypothesis (Fig. 1). Under the unguarded $\mathrm{X}$ hypothesis, outbred male F1 offspring of the shorter-lived maternal line inherit deleterious mutations on their X chromosome, resulting in lower lifespan than offspring from the longer-lived maternal line without recessive deleterious mutations on the $\mathrm{X}$ chromosome. Although the effects of recessive deleterious mutations may be underestimated in crosses between highly inbred lines due to higher expected levels of purging during the inbreeding process (Hedrick, 1994), similar to studies in D. melanogaster (Brengdahl et al., 2018a), the unguarded $\mathrm{X}$ hypothesis is appears to be insufficient to explain sexual dimorphism in D. serrata lifespan. Sex-specific differences in selection (Bonduriansky et al., 2008,

211 Maklakov et al., 2009, Maklakov and Lummaa, 2013) could better explain the pattern of higher

212 mortality in males and lifespan dimorphism observed in D. serrata. Alternative explanations

213 that partly explain the patterns predicted by the unguarded X hypothesis and could be explored 214 in future studies include sexually antagonistic genes and sex-specific expression patterns

215 (Sultanova et al., 2018, Brengdahl et al., 2018a). 
219 In addition to sex- and genotype-biased longevity, we also found interactions of genotype with

220 mating and density, our two experimentally manipulated axes of social background. Across a range of taxa, sexual dimorphism is a result of complex relationships between environmental conditions and sex-specific reproductive costs (Lemaitre et al., 2020). Mean lifespans did not differ significantly between density treatments within genotypes (Fig. 2), even though large sex and genotype effects were detected. While we detected no Genotype $\times$ Density or Sex $\times$ Density interaction, there was a highly significant interaction between density and mating that appeared to be driven by a change in rank order lifespan between low and medium density, which was highest for low density in the mated treatment but lowest for the unmated treatment (Fig. 2). Survivorship experiments with high densities at the beginning can produce high mortality rates at young ages (Graves and Mueller, 1993), however we observed no such effect in our high density treatments.

In our study, mating had no effect on mean lifespan in D. serrata. While we did detect a significant Genotype $\times$ Mating interaction this can be explained by idiosyncratic effects of genotype on mating and density (Fig 1). Adverse effects of multiple mating on lifespan in $D$. melanogaster males have been reported in several studies, as have toxic effects of male accessory gland proteins on female fitness and lifespan (Fowler and Partridge, 1989, Chapman et al., 1995). In female D. serrata, continued male courtship and harassment also leads to decreased fitness in females (Chenoweth et al., 2015). Intermittent and short-term mating, as was the case in this study, could explain why mated and unmated flies have similar lifespans, except at low density in two genotypes where unmated flies lived on average 6 days longer. Though widespread, trade-offs between longevity and reproduction are hardly ubiquitous, can be highly plastic, and uncoupled under certain environmental or genetic conditions (Flatt, 2011). 
Conclusion

248 Here, we show that the pattern of sexual dimorphism in D. serrata is consistent with females

249 living longer than males across all genotypes and treatments. As expected, outbred genotypes 250 lived longer, and female lifespan was more adversely affected by inbreeding. However outbred 251 male lifespan for the outbred F1 genotypes did not differ as expected from a cross between 252 parental genotypes with significantly different lifespans. Overall, our findings converge with existing evidence to suggest that sex-specific selection largely drives the sexual dimorphism seen in lifespan (Bonduriansky et al., 2008, Maklakov et al., 2009, Maklakov and Lummaa, 2013), and that physiological differences resulting from strategies developed amongst sexes to maximize fitness can be independent of the effects of mating and/or density (Sultanova et al., 2020, Maklakov et al., 2017, Harvanek et al., 2017, Kimber and Chippindale, 2013, Ziehm et al., 2013, Vermeulen and Bijlsma, 2004a, Vermeulen and Bijlsma, 2004b). As the first study dissecting contributions of genetic background and social environment on lifespan in $D$. serrata, the robustness of these findings will no doubt be revealed by further testing effects on lifespan across different conditions. It is however reasonable to conclude that, based on a variety of studies across different taxa and Drosophila species, ageing in D. serrata is best viewed as a condition-dependent environmental modulation of a genetically determined trait.

Acknowledgements

266 We thank Nicholas Appleton for assistance with fly work.

\section{Competing interests}

268 The authors declare no competing financial interests.

\section{Author Contributions}

271 All authors contributed to the planning of the experiments; V.P.N performed the experiments and 272 the other authors assisted in analyses, interpretation, and writing the manuscript. Funding

273 This research was supported with funding provided by the University of Queensland. V.P.N.

274 was supported by the QUEX Institute - a joint initiative of The University of Queensland and 275 the University of Exeter. 
References

AIGAKI, T. \& OHBA, S. 1984. Effect of mating status on Drosophila virilis lifespan. Experimental Gerontology, 19, 267-278.

AUSTAD, S. N. 2019. Sex differences in health and aging: a dialog between the brain and gonad? Geroscience, 41, 267-273.

BALLARD, J. W. O. 2005. Drosophila simulans as a novel model for studying mitochondrial metabolism and aging. Experimental Gerontology, 40, 763-773.

BONDURIANSKY, R., MAKLAKOV, A., ZAJITSCHEK, F. \& BROOKS, R. 2008. Sexual selection, sexual conflict and the evolution of ageing and life span. Functional Ecology, 22, 443-453.

BRENGDAHL, M., KIMBER, C. M., MAGUIRE-BAXTER, J. \& FRIBERG, U. 2018a. Sex differences in life span: Females homozygous for the X chromosome do not suffer the shorter life span predicted by the unguarded X hypothesis. Evolution, 72, 568-577.

BRENGDAHL, M., KIMBER, C. M., MAGUIRE-BAXTER, J., MALACRINO, A. \& FRIBERG, U. 2018b. Genetic Quality Affects the Rate of Male and Female Reproductive Aging Differently in Drosophila melanogaster. Am Nat, 192, 761-772.

BURGER, J. M. \& PROMISLOW, D. E. 2004. Sex-specific effects of interventions that extend fly life span. Sci Aging Knowledge Environ, 2004, pe30.

CARAZO, P., GREEN, J., SEPIL, I., PIZZARI, T. \& WIGBY, S. 2016. Inbreeding removes sex differences in lifespan in a population of Drosophila melanogaster. Biol Lett, 12.

CHAPMAN, T., LIDDLE, L. F., KALB, J. M., WOLFNER, M. F. \& PARTRIDGE, L. 1995. Cost of mating in Drosophila melanogaster females is mediated by male accessory gland products. Nature, 373, 241-244.

CHENOWETH, S. F., APPLETON, N. C., ALLEN, S. L. \& RUNDLE, H. D. 2015. Genomic Evidence that Sexual Selection Impedes Adaptation to a Novel Environment. Curr Biol, 25, 1860-6.

FLATT, T. 2011. Survival costs of reproduction in Drosophila. Exp Gerontol, 46, 369-75.

FOWLER, K. \& PARTRIDGE, L. 1989. A cost of mating in female fruitflies. Nature, 338, 760-761.

FRIBERG, U. 2005. Genetic variation in male and female reproductive characters associated with sexual conflict in Drosophila melanogaster. Behav Genet, 35, 455-62.

GRAVES, J. L., JR. \& MUELLER, L. D. 1993. Population density effects on longevity. Genetica, 91, 99-109.

HARVANEK, Z. M., LYU, Y., GENDRON, C. M., JOHNSON, J. C., KONDO, S., PROMISLOW, D. E. L. \& PLETCHER, S. D. 2017. Perceptive costs of reproduction drive ageing and physiology in male Drosophila. Nat Ecol Evol, 1, 152.

HEDRICK, P. W. 1994. Purging inbreeding depression and the probability of extinction: fullsib mating. Heredity (Edinb), 73 ( $\mathrm{Pt}$ 4), 363-72.

ILIADI, K. G., ILIADI, N. N. \& BOULIANNE, G. L. 2009. Regulation of Drosophila lifespan: effect of genetic background, sex, mating and social status. Exp Gerontol, 44, 546-53.

JOSHI, A. \& MUELLER, L. D. 1997. Adult crowding effects on longevity in Drosophila melanogaster: Increase in age-independent mortality. Current Science, 72, 255-260.

KHAZAELI, A. A., XIU, L. \& CURTSINGER, J. W. 1995. Effect of adult cohort density on age-specific mortality in Drosophila melanogaster. J Gerontol A Biol Sci Med Sci, 50, B262-9.

KHAZAELI, A. A., XIU, L. \& CURTSINGER, J. W. 1996. Effect of density on age-specific mortality in Drosophila: a density supplementation experiment. Genetica, 98, 21-31. 
KIMBER, C. M. \& CHIPPINDALE, A. K. 2013. Mutation, condition, and the maintenance of extended lifespan in Drosophila. Curr Biol, 23, 2283-2287.

KOLIADA, A., GAVRILYUK, K., BURDYLYUK, N., STRILBYTSKA, O., STOREY, K. B., KUHARSKII, V., LUSHCHAK, O. \& VAISERMAN, A. 2020. Mating status affects Drosophila lifespan, metabolism and antioxidant system. Comp Biochem Physiol A Mol Integr Physiol, 246, 110716.

MAKLAKOV, A. A., BONDURIANSKY, R. \& BROOKS, R. C. 2009. Sex differences, sexual selection, and ageing: an experimental evolution approach. Evolution, 63, 2491-503.

MAKLAKOV, A. A., CARLSSON, H., DENBAUM, P., LIND, M. I., MAUTZ, B., HINAS, A. \& IMMLER, S. 2017. Antagonistically pleiotropic allele increases lifespan and late-life reproduction at the cost of early-life reproduction and individual fitness. Proc Biol Sci, 284.

MAKLAKOV, A. A. \& LUMMAA, V. 2013. Evolution of sex differences in lifespan and aging: causes and constraints. Bioessays, 35, 717-24.

MALICK, L. E. \& KIDWELL, J. F. 1966. The effect of mating status, sex and genotype on longevity in Drosophila melanogaster. Genetics, 54, 203-9.

PIPER, M. D. W. \& PARTRIDGE, L. 2018. Drosophila as a model for ageing. Biochim Biophys Acta Mol Basis Dis, 1864, 2707-2717.

REDDIEX, A. J., ALLEN, S. L. \& CHENOWETH, S. F. 2018. A Genomic Reference Panel for Drosophila serrata. G3 (Bethesda), 8, 1335-1346.

ROBSON, S. K., VICKERS, M., BLOWS, M. W. \& CROZIER, R. H. 2006. Age determination in individual wild-caught Drosophila serrata using pteridine concentration. $J$ Exp Biol, 209, 3155-63.

ROGINA, B. 2011. For the special issue: aging studies in Drosophila melanogaster. Exp Gerontol, 46, 317-9.

SERVICE, P. M. 1989. The effect of mating status on lifespan, egg laying, and starvation resistance in Drosophila melanogaster in relation to selection on longevity. Journal of Insect Physiology, 35, 447-452.

SULTANOVA, Z., ANDIC, M. \& CARAZO, P. 2018. The "unguarded-X" and the genetic architecture of lifespan: Inbreeding results in a potentially maladaptive sex-specific reduction of female lifespan in Drosophila melanogaster. Evolution, 72, 540-552.

SULTANOVA, Z. \& CARAZO, P. 2019. Sex ratio at mating does not modulate age fitness effects in Drosophila melanogaster. Ecol Evol, 9, 6501-6507.

SULTANOVA, Z., GARCIA-ROA, R. \& CARAZO, P. 2020. Condition dependent mortality exacerbates male (but not female) reproductive senescence and the potential for sexual conflict. J Evol Biol.

SWINDELL, W. R. \& BOUZAT, J. L. 2006. Selection and inbreeding depression: effects of inbreeding rate and inbreeding environment. Evolution, 60, 1014-22.

TAN, C. K., PIZZARI, T. \& WIGBY, S. 2013. Parental age, gametic age, and inbreeding interact to modulate offspring viability in Drosophila melanogaster. Evolution, 67, 3043-51.

TRIVERS, R. 1985. Social evolution.

VAISERMAN, A. M., ZABUGA, O. G., KOLYADA, A. K., PISARUK, A. V. \& KOZERETSKA, I. A. 2013. Reciprocal cross differences in Drosophila melanogaster longevity: an evidence for non-genomic effects in heterosis phenomenon? Biogerontology, 14, 153-63.

VERMEULEN, C. J. \& BIJLSMA, R. 2004a. Changes in mortality patterns and temperature dependence of lifespan in Drosophila melanogaster caused by inbreeding. Heredity (Edinb), 92, 275-81. 
VERMEULEN, C. J. \& BIJLSMA, R. 2004b. Characterization of conditionally expressed mutants affecting age-specific survival in inbred lines of Drosophila melanogaster: lethal conditions and temperature-sensitive periods. Genetics, 167, 1241-8. Drosophila species: a comparative study on life span, environmental variables and stress resistance. J Evol Biol, 28, 1892-900.

XIROCOSTAS, Z. A., EVERINGHAM, S. E. \& MOLES, A. T. 2020. The sex with the reduced sex chromosome dies earlier: a comparison across the tree of life. Biol Lett, 16, 20190867.

ZAJITSCHEK, F., ZAJITSCHEK, S. R., FRIBERG, U. \& MAKLAKOV, A. A. 2013. Interactive effects of sex, social environment, dietary restriction, and methionine on survival and reproduction in fruit flies. Age, 35, 1193-1204.

ZIEHM, M., PIPER, M. D. \& THORNTON, J. M. 2013. Analysing variation in Drosophila aging across independent experimental studies: a meta-analysis of survival data. Aging Cell, 12, 917-22. 


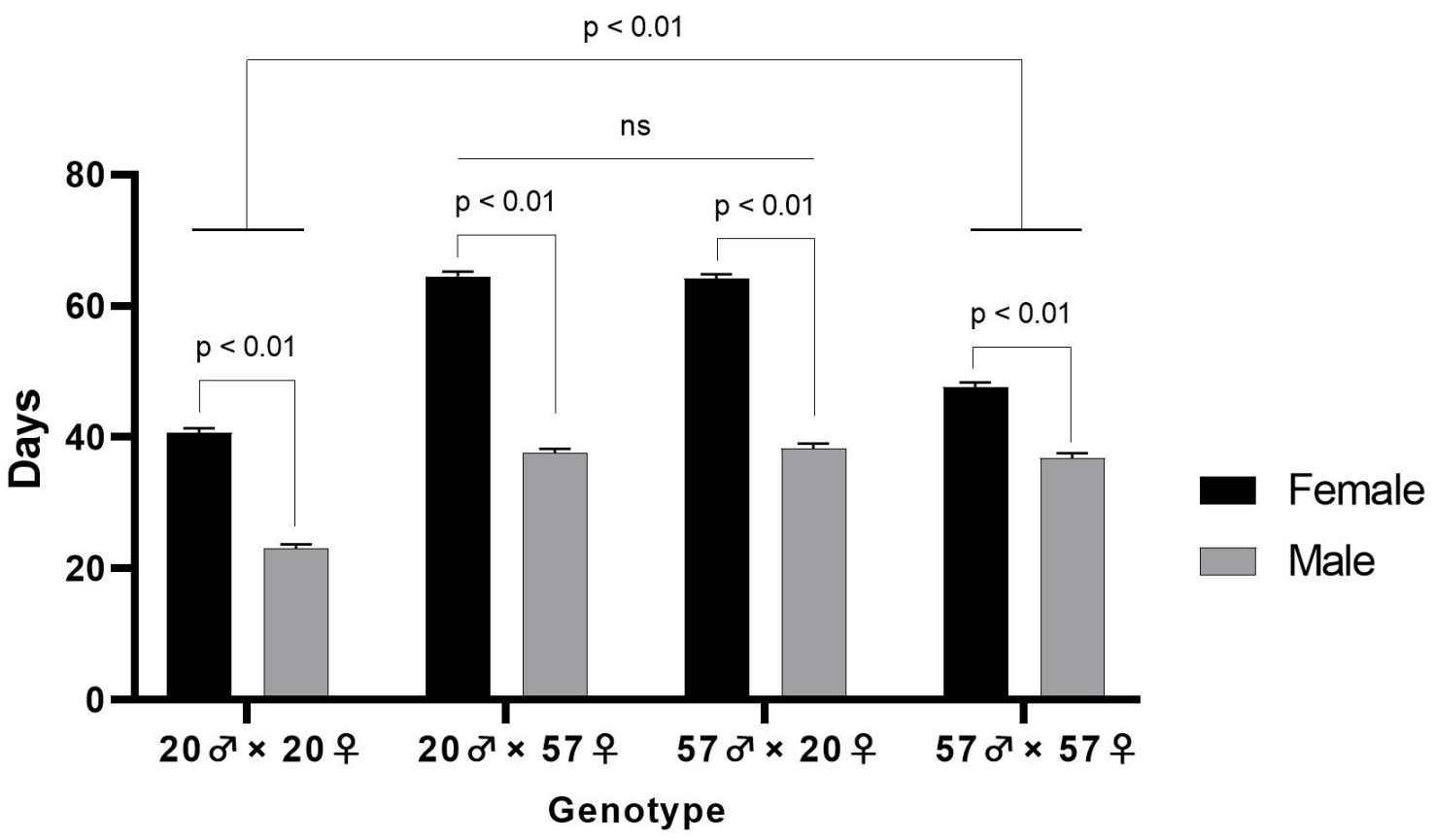

394 Figure 1. Genotype dependent effects on sex differences in lifespan in D. serrata. Sex

395 differences in mean adult life span in the four genotypes resulting for our reciprocal cross

396 between DsGRP20 and DsGRP57 (two parental lines plus alternate F1s). Bars represent the

397 mean lifespan of each genotype pooled across the six density (low, medium, and high) $\times$ mating

398 status (mated and non-mated) treatment combinations. Error bars represent $1 \mathrm{~S}$. E. 


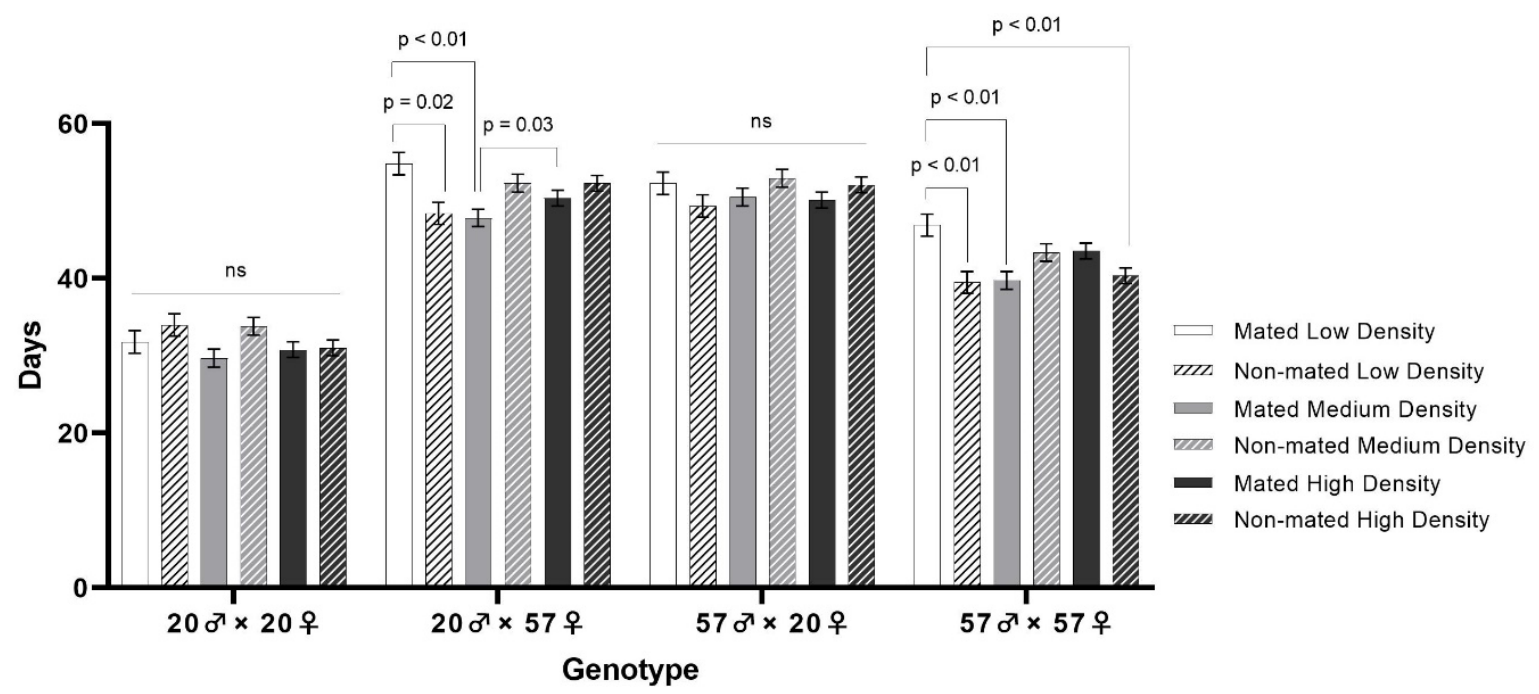

401

402 Figure 2. Genotype dependent effects of the social environment on lifespan in D. serrata.

403 Shown are pooled adult, male and female lifespan for the homozygous founder lines

$404 D s G R P 200^{7} \times D s G R P 20 \%$ and DsGRP57 $0^{7} \times D s G R P 57 \%$, as well as both reciprocal F1

405 crosses DsGRP20 O" $\times$ DsGRP57 $q$ and DsGRP57 $0^{\prime \prime} \times D s G R P 20 \%$ between these lines.

406 Each bar represents the mean of each genotype measured in one of six different density (low,

407 medium, and high) $\times$ mating status (mated and non-mated) treatment combinations Error bars

408 represent the 1 S.E. of the mean.

409 
410 Tables

411 Table 1. F-tests of fixed effects for the reduced model examining the significance of

412 contributions of sex, genotype, mating, and density to $D$. serrata lifespan.

413

\begin{tabular}{|c|c|c|c|}
\hline Effect & d.f. & $\boldsymbol{F}$ & $\boldsymbol{P}$ \\
\hline Sex & $1,454.4$ & 1798.3 & $4.54 \mathrm{e}^{-160}$ \\
\hline Genotype & $3,601.3$ & 340.4 & $3.83^{\mathrm{e}-129}$ \\
\hline Sex $\times$ Genotype & $3,454.3$ & 64.6 & $8.36^{\mathrm{e}-35}$ \\
\hline Density & $2,552.9$ & 1.18 & 0.308 \\
\hline Genotype $\times$ Density & $6,552.7$ & 0.84 & 0.539 \\
\hline Mating & $1,601.5$ & 0.09 & 0.764 \\
\hline Genotype $\times$ Mating & $3,601.3$ & 3.28 & 0.021 \\
\hline Density $\times$ Mating & $2,552.9$ & 15.0 & $4.53^{\mathrm{e}-07}$ \\
\hline Genotype $\times$ Density $\times$ Mating & $6,552.8$ & 2.45 & 0.024 \\
\hline
\end{tabular}

